\title{
Re-positive of SARS-CoV-2 test is common in COVID-19 patients after hospital discharge. Data from high standard post-discharge quarantined patients in Macao SAR, China
}

\author{
Chi Leong Wong ${ }^{\text {Equal first author, } 1}$, Sao Kuan Lei ${ }^{\text {Equal first author, } 1}{ }^{\text {, Chin Ion Lei }}{ }^{\text {Corresp., }}{ }^{2}$, Iek Long Lo ${ }^{3}$, Chong Lam ${ }^{4}$, Iek Hou \\ Leong ${ }^{4}$ \\ ${ }^{1}$ Macao Academy of Medicine, Centro Hospitalar Conde de São Januário, Health Bureau, Macao SAR, China \\ 2 Department of Medicine, Centro Hospitalar Conde de São Januário, Health Bureau, Macao SAR, China \\ 3 Department of Respiratory Medicine, Centro Hospitalar Conde de São Januário, Health Bureau, Macao SAR, China \\ ${ }^{4}$ Center for Disease Control and Prevention, Health Bureau, Macao SAR, China \\ Corresponding Author: Chin Ion Lei \\ Email address: cilei@ssm.gov.mo
}

During the pandemic of COVID-19, Macau faces tremendous pressure because she is a famous gambling and tourism city with the world's highest population density. Macau government implemented decisive public health intervention to control the transmission of COVID-19, and there were only two independent outbreaks in Macau. In the second outbreak, all 35 cases were infected in foreign countries. They were quarantined in airborne infection isolation rooms for at least 14 days with reverse transcriptionpolymerase chain reaction (RT-PCR) tests after hospital discharge. 12 (34.3\%) of them had re-positive SARS-CoV-2 test results, and none of them presented any COVID-19 signs or symptoms during the entire quarantine period. In this study, the re-positive patients were more likely to be diagnosed in the early stage of the disease with a longer hospital stay. Virus re-infection is impossible in this high standard isolation setting, and reactivation is also unlikely, so that residual virus nucleic acid should be the possible reason for this phenomenon. Due to limited data about the risk of re-positive patients, it is better to quarantine patients after discharge for a prolonged period with repeat RT-PCR tests to minimize the community's potential risk, particularly in the regions with relative plenty of resources and low community infection rate such as Macau. 
2 Re-positive of SARS-CoV-2 test is common in COVID-

319 patients after hospital discharge. Data from high

4 standard post-discharge quarantined patients in

5 Macao SAR, China

6

7

8

9

10

11

12

13

14

15

16

17

18

19

20

21

22

23

Chi Leong Wong Equal first author, 1, Sao Kuan Lei Equal first author, 1, Chin Ion Lei Corresp., 2, Iek Long Lo

${ }^{3}$, Chong Lam ${ }^{4}$, Iek Hou Leong ${ }^{4}$

${ }^{1}$ Macao Academy of Medicine, Centro Hospitalar Conde de São Januário, Health Bureau, Macao SAR, China

${ }^{2}$ Department of Medicine, Centro Hospitalar Conde de São Januário, Health Bureau, Macao

SAR, China

${ }^{3}$ Department of Respiratory Medicine, Centro Hospitalar Conde de São Januário, Health Bureau, Macao SAR, China

${ }^{4}$ Center for Disease Control and Prevention, Health Bureau, Macao SAR, China

Corresponding Author:

Dr. Chin Ion Lei ${ }^{2}$

Department of Internal Medicine, Centro Hospitalar Conde de São Januário, Health Bureau, Estrada do Visconde de S. Januário, Macao SAR, China.

Email address: cilei@ssm.gov.mo 


\section{Abstract}

25 During the pandemic of COVID-19, Macau faces tremendous pressure because she is a famous gambling and tourism city with the world's highest population density. Macau government implemented decisive public health intervention to control the transmission of COVID-19, and

28

29

30

31

32

33

34

35

36

37

38

39

40

41

42

43

44

45

46

47

48

49

50

51

52

53

54

55

56

57

58

59

60

61

62

63

there were only two independent outbreaks in Macau. In the second outbreak, all 35 cases were infected in foreign countries. They were quarantined in airborne infection isolation rooms for at least 14 days with reverse transcription-polymerase chain reaction (RT-PCR) tests after hospital discharge. $12(34.3 \%)$ of them had re-positive SARS-CoV-2 test results, and none of them presented any COVID-19 signs or symptoms during the entire quarantine period. In this study, the re-positive patients were more likely to be diagnosed in the early stage of the disease with a longer hospital stay. Virus re-infection is impossible in this high standard isolation setting, and reactivation is also unlikely, so that residual virus nucleic acid should be the possible reason for this phenomenon. Due to limited data about the risk of re-positive patients, it is better to quarantine patients after discharge for a prolonged period with repeat RT-PCR tests to minimize the community's potential risk, particularly in the regions with relative plenty of resources and low community infection rate such as Macau.

\section{Introduction}

In December 2019, a series of pneumonia cases of novel coronavirus infection emerged in Wuhan city of Hubei province in China. Then, the disease spread quickly and widely worldwide and was named COVID-19. ${ }^{1}$ As a rising number of the issue, the WHO declared the outbreak as a Public Health Emergency of International Concern on $30 \mathrm{Jan} 2020 .^{2} \mathrm{Up}$ to early November, the accumulative number of confirmed cases has reached over 46 million worldwide, and it continues to rise. ${ }^{3}$ Some studies even found the Spike D614G mutation of the virus with increased infectivity in other countries while comparing the virus samples in China. ${ }^{4}$ As a famous gambling and tourism city with the highest population density globally, Macau faces massive stress from overseas and local populations during the coronavirus pandemic. From our point of view, Macau fights against COVID-19 quite successfully. There were only 45 confirmed cases in Macau's two irrelevant outbreaks before late June, without local transmission. All ten patients in the first outbreak from 22 Jan to 4 Feb were from Mainland China, while the 35 cases in the second episode from 15 Mar to 8 Apr came from foreign countries. After that, there was no more new case for over two months. ${ }^{5}$ The Macau Government's tremendous effort contributed to the great success in this fighting.

We early upgraded our port policy to a 14-d quarantine with at least twice RT-PCR tests of novel coronavirus for all people who came from other countries since March. ${ }^{6}$ On the other hand, we had a loose community testing policy, making RT-PCR tests accessible to everyone whenever they requested. Under these aggressive public health strategies, we could safely confirm all infected cases in the second outbreak.

Meanwhile, studies reported SARS-CoV-2 re-positive cases after the patients fulfilled discharge criteria. ${ }^{7-10}$ It raised concerns about the virus's re-infection or reactivation, regardless of whether

Peer] reviewing PDF | (2020:11:55715:1:1:NEW 3 Feb 2021) 
64 there was no substantial evidence about their infectivity. As viewing this, we quarantined all

65

66

67

68

69

70

71

72

73

\section{4}

75

76

77

78

79

80

81

82

83

84

85

86

87

88

89

90

91

92

93

94

95

96

97

98

99

100

101

102

103

patients who fulfilled discharge criteria in airborne infection isolation rooms, for at least 14 days, with RT-PCR test on day 10 and day 13, before they were back to the Macau community. The re-positive rate in academic reports was 3.3\% to $21.4 \% \cdot{ }^{8-13}$ Most of their patients got infected in China. Some were home isolated while some used inconsistent novel coronavirus test methods and other surveys represented only partial cases in a city. Here, we reported the repositive rate of all COVID-19 patients in Macau during the second outbreak. All of them were infected outside of China and observed under a high standard quarantine location with repeated nasopharyngeal swab RT-PCR tests after hospital discharge.

\section{Materials \& Methods}

Study design

This retrospective study included all COVID-19 confirmed cases $(n=35)$ in Macau during the second independent outbreak which was from $15 \mathrm{Mar}, 2020$ to $8 \mathrm{Apr}$, 2020. They were from foreign countries and were admitted to Centro Hospitalar Conde de São Januário (CHCSJ), Macau SAR, China. We discharged the patients when they fulfilled the criteria of the $7^{\text {th }}$ edition from the National Health Commission of China, which included obvious improved symptoms and pulmonary imaging and at least twice consecutively negative RT-PCR tests on nasopharyngeal samples. ${ }^{14}$ Then, the patients were transferred to a standard isolation ward for at least 14 days for post-discharge quarantine. Experienced medical staff closely observed them and performed two times RT-PCR assay on their nasopharyngeal specimen on day 10 and day 13 of quarantine. The negative group was the cases with negative results in all RT-PCR tests, while repositive patients were the ones who had at least one positive RT-PCR test during the quarantine. Before returning to the community, the re-positive subjects had to repeat the test every other day until three consecutive negative results. The Hospital Medical Ethical Committee of Centro Hospitalar Conde de São Januário, Macao SAR, China approved this study (Ethical Application Ref: 0044/MEC/N/2020). The committee waived the requirement for patient consent.

\section{Data collection}

We collected the data from both electric and written medical records. It consisted of demographic data, epidemiological data, clinical data, including severity classifications, length of stay, symptoms, signs, laboratory results, radiological results, main treatment strategies, and post-discharge quarantine data.

\section{Standard isolation ward}

There were 60 airborne infection isolation rooms (AIIR) in our quarantine center with up to two beds per room, but only minors could share space with the family members. Each room had an anteroom, electric self-closing doors with an interlocking system, clean to dirty airflow, negative pressure monitor, with at least 12 air exchanges per hour, and high-efficiency particulate air filtration, which fulfilled American Centers for Disease Control and Prevention suggestion. ${ }^{15}$ 
104 Furthermore, qualified doctors and nurses were working in the ward 24 hours a day. All staff

105

106

107

108

109

110

111

112

113

114

115

116

117

118

119

120

121

122

123

124

125

126

127

128

129

130

131

132

133

134

135

136

137

138

139

140

141

142

143

used personal protective equipment (PPE), including gloves, isolation gown, N95 mask, face shield, headgear, and foot cover when they entered AIIR, and discarded them properly at once when they left the room to avoid cross-infection.

Reverse Transcriptase-Polymerase Chain Reaction (RT-PCR) test for SARS-CoV-2

Nasopharyngeal swab (NPS) samples were tested using a commercial SARS-CoV-2 ORF1ab/N Gene Nucleic acid detection kit (BioGerm, China) following the manufacturer's instructions. A cycle threshold value (Ct-value) less than or equal to 35 was defined as a positive test result.

Statistical analysis

SPSS ver. 25.0 (SPSS Inc., Chicago, IL, USA) conducted the statistical analyses. Continuous data were presented as median \pm interquartile range (IQR); categorical variables were presented as frequency/percentage. For continuous variables, the Mann-Whitney U test was used for intergroup comparisons among groups with skewed distributions. For categorical variables, the chi-square test or Fisher's exact test was used. A two-sided significance level of 0.05 was used to evaluate statistical significance.

\section{Results}

Re-positive rate and clinical characteristics of the re-positive group

There were 35 confirmed cases in this study, and 12 (34.3\%, 95\%CI 21.5\%-49.3\%) were found to be re-positive during the post-discharge quarantine in AIIR. Both males and females had six people, and the median age was 28.5 years old (IQR: 18.3-42.3). Serum IgG antiviral antibody was positive in all patients before the quarantine. The median days between the re-positive NPS sample and IgG antibody detection was 30 days (IQR: 19.3-44). Furthermore, none of the cases had any signs or symptoms of COVID-19 during the entire quarantine period.

Risk factor analysis

Age and gender: The median age of the negative group was 28 years old (IQR: 20-44), like the re-positive group. There were seventeen males and six females in the negative group, with a $26.1 \%$ re-positive rate in male patients and $50 \%$ in females. There were no statistically significant differences in age or gender between the re-positive and negative groups (Tab 1, p > $0.05)$.

Nationality and travel history: Most of the patients were Chinese $(n=23,65.7 \%)$ and back from England $(\mathrm{n}=16,45.7 \%)$. The re-positive rate in Filipino $(\mathrm{n}=3)$ and Korean $(\mathrm{n}=1)$ were $100 \%$ while Chinese was $34.8 \%$, Indonesian $(n=3)$ and Portuguese $(n=3)$ was $0 \%$, closed to being statistically significant (Tab $1, \mathrm{p}=0.066$ ). For travel history, re-positive rate had no statistically significant differences between different groups (Tab $1, p>0.05$ ).

Clinical condition: Signs and symptoms, clinical classification, past medical history, laboratory test, computed tomography, and therapeutic schedules were compared between the two groups.

PeerJ reviewing PDF | (2020:11:55715:1:1:NEW 3 Feb 2021) 
144 None of them had significant differences (Tab $1 \&$ Tab 2, p > 0.05). Nevertheless, we noticed

145 that those clinically classified as asymptomatic types had no re-positive results. Simultaneously,

146 those with some signs or symptoms before hospitalization were more likely to be re-positive

147 (Tab 1, 39.3\% vs. 14.3\%).

148 Timeline of disease progression: We analyzed the duration between the onset of symptoms or 149 signs (S/S), diagnosis, and the date of hospital discharge criteria fulfilled. There were seven 150 patients without any $\mathrm{S} / \mathrm{S}$ before admission. In the re-positive group, the median days from $\mathrm{S} / \mathrm{S}$ 151 onset to diagnosis was shorter (2 days vs. 4 days) while the median days from $\mathrm{S} / \mathrm{S}$ onset to 152 hospital discharge and from diagnosis to hospital discharge were longer (43 days vs. 35 days \&

15338 days vs. 27 days). However, no significant difference was found in these parameters between 154

155

\section{Discussion}

157

158

159

160

161

162

163

164

165

166

167

168

169

170

171

172

173

174

175

176

177

178

179

180

181

182

One-third of patients had re-positive SARS-CoV-2 test results in our study, which is higher than other studies. ${ }^{8-13}$ Under the outstanding public health strategies and an ideal environment in Macau, we could evaluate the re-positive rate of all the patients quarantined in a high standard location with RT-PCR tests after hospital discharge within one independent outbreak. After all the serious intervention and observation, we could conclude that a significant proportion of discharged patients in Macau were carriers of the virus nucleic acid. Because they were all imported cases in our study, we suspected this phenomenon might be shared globally. The re-positive rate in our study was as high as $34.3 \%$. A more significant number of tests may be one possible reason. We performed twice tests while the other reported countries only had once. ${ }^{9-13}$ The probability of obtaining a false-negative result increased with time from symptom onset and could be drastically reduced by repeat testing. ${ }^{16}$ In previous studies, the heterogenic design, case selection, and various sampling sites and frequency led to a relatively doubtful repositive rate. ${ }^{8-13}$ In contrast, our result should provide more accurate data since we assessed all cases in an outbreak with a unified approach.

Apart from false-negative results, false-positive tests should be considered another likely reason for the inaccurate re-positive rate. The primary route of false-positive PCR test is contamination, including cross-contamination between specimens or synthetically derived target nucleic acids. ${ }^{17}$ All our specimens were performed in an ISO 15189 accredited medical laboratory. Therefore, the likelihood of contamination should be low, and subsequently the false-positive cases. Along with the global dispersal of COVID-19, mutant viruses have emerged and lead to alteration of the virus behavior. The Spike protein amino acid change D614G is one of the most concerned SARS-CoV-2 variants, which has become apparent in Europe and rapidly replacing other versions of the virus globally since late February, except in China. ${ }^{4}$ Different from most existing research, all cases in this study were from foreign countries and might be infected by the D614G variant. One recent research found that the D614G replacement was associated with higher viral loads. ${ }^{18}$ It can be an explanation of the unexpected high re-positive rate. 
183 Our study showed a trend that symptomatic patients were more likely to be re-positive. It also 184 found that the median days from $\mathrm{S} / \mathrm{S}$ onset to diagnosis was shorter in the re-positive group than 185 the negative group, but the duration from diagnosis to hospital discharge was the opposite.

186 However, their statistical significance could not be evaluated due to the small sample size. This

187

188

189

190

191

192

193

194

195

196

197

198

199

200

201

202

203

204

205

206

207

208

209

210

211

212

213

214

215

216

217

218

219

220

221

222 result reflected that re-positive patients were more likely to have an earlier diagnosis and more extended hospitalization than the negative group. In other words, the symptomatic and early diagnosed patients tended to be re-positive during the quarantine period. Can there be a higher virus load in vivo cells in this group of patients, with more pronounced symptoms and signs that make a more straightforward diagnosis? Further study is worth to clear this issue.

Although several studies analyzed the risk factors of recurrent positive SARS-CoV-2 test among discharged patients, ${ }^{8-10,12,13}$ there was no unanimous conclusion till the present moment. Some research found the re-positive result related to different risk factors such as age, symptoms, illness severity, laboratory tests, etc. Still, they had the opposite contribution in other reports. Indeed, none of the present studies could discover reliable indicators to forecast the patient's risk of being re-positive SARS-CoV-2. Our analysis did not establish any risk factors in terms of demographic or clinical characteristics, laboratory examination, or treatment strategies. As a high re-positive rate was noted and failure to discover risk factors, should re-positive be a natural pathophysiological process of COVID-19?

Except for laboratory error, the causes of a re-positive SARS-CoV-2 test result after hospital discharge have several assumptions. They are re-infection, reactivation, and residual virus nucleic acid.

Hong Kong experts confirmed the possibility of re-infection in COVID-19 patients in a recent study. ${ }^{19}$ It could be one reason for the re-positive test in the previous academic reports. Unclarified or home isolation strategy could imply a little or some opportunity for the people to be exposed to the infection source again. Our research quarantined all patients in airborne infection isolation rooms and under management by professional medical staff with suitable personal protective equipment. Meanwhile, there was no infection report in medical staff and no local infection case in Macau. As a result, we can exclude the chance of re-infection in this high standard environment.

Reactivation is unlikely since all our re-positive cases were asymptomatic during post-discharge quarantine, and they already had IgG antiviral antibodies in serum samples before discharge.

After deducting the above hypotheses, residual virus nucleic acid in vivo cell is a possible cause of the re-positive phenomenon.

Liu WD and colleagues reported a COVID-19 case with prolonged virus shedding even after seroconversion. ${ }^{20}$ It implied that the SARS-CoV-2 could exist in human cells in vivo for an extended period. On the other hand, Justin W and colleagues observed the oscillation of positive and negative SARS-CoV-2 NPS test results in the patients, which related to a fluctuating cycle threshold $(\mathrm{Ct})$ value. Therefore, we presume virus load would descend along with the disease's recovery, with a relative up and down pattern rather than in a straight line. When infected cells were intermittently shedding at the level above and below the detection limit, conversion 
223 between positive and negative results would appear. Once we conducted the test at the point of 224 fewer shedding cells, the result would be negative, and the patient would fulfill the discharge

225

226

227

228

229

230

231

232

233

234

235

236

237

238

239

240

241

242

243

244

245

246

247

248

249

250

251

252

253

254

255

256

257

258

259

260

261

262 criteria. Actually, the virus shedding load just hit the detection limit, and more cells would be passed at the other time, then the patient would have a re-positive test result. Of course, further precise and quantitative experimental studies are needed to verify this hypothesis.

It is crucial to clarify the infectivity in re-positive patients clinically and in public health decision making. However, it remains unknown despite substantive reports and research. The virus's ability to replicate in cultured cells serves as a surrogate marker of infectivity, but we did not perform virus culture due to technology limitations. On the other hand, culture is less sensitive than RT-PCR for detection of live viruses. Even with the culture of virus, mixed results were reported in different studies. Wölfel $\mathrm{R}$ and colleagues found that no virus could be cultured in patient samples after day 9 of symptom onset. ${ }^{21}$ In contrast, Liu WD and colleagues reported isolated SARS-CoV-2 from the sputum sample on day 18 after symptoms onset. ${ }^{20}$ Therefore, there is still no applicable clinical method to establish SARS-CoV-2's infectivity. The potential risk of virus transmission from a re-positive patient cannot be eliminated.

In Macau, most of our citizens are susceptible to COVID-19 since there is no local epidemic, and only very few residents were infected. Fortunately, enough resources such as AIIR, PPE, healthcare human resources, etc., which allow high quality and prolonged quarantine and minimize transmission risk in our city. After balancing the risk and benefit, we released repositive patients from quarantine after three consecutive negative results. Zou Y and colleagues also supported that patients with three consecutive negative results had a much lower recurrent positive rate than two negative findings. ${ }^{9}$ This public health strategy contributes to Macau's achievements of beating back COVID-19. However, it did bring some undesired experience to the individuals. For example, prolonged isolation in a health facility is prone to psychological problems. As a result, other cities may not simply copy our methods. The health authority should make their decision according to community acceptance ability. Nonetheless, we agreed that quarantine at home for two weeks with PCR tests after hospital discharge is the least transmission prevention since SARS-CoV-2 is a novel virus with only scanty knowledge about it and no effective treatment until now.

Lastly, some limitations of this study should be noted. First, the sample size was relatively small. There were only 35 cases in our study, even though all patients in Macau's second outbreak had been included. Second, the onset time and the presentation of sign or symptom was according to the patient's statement so that recall bias may present in the timeline of disease progression. Third, we did not perform the virus culture, and the infectivity could not be shown. Fourth, we could not get $\mathrm{Ct}$ values and genomic sequencing of SARS-CoV-2 due to laboratory policy. They should be considered in future studies.

According to the global COVID-19 situation, the disease seems to keep coexisting with humans for a long time. Since a high re-positive rate among COVID-19 patients was presented in our study, we can imagine that there will be a great practical challenge to handle this group of patients. Different underlying causes had different approaches. How can we distinguish re- 
263 infection, reactivation, and residual virus nucleic acid from each other? To do or not to do the 264 whole-genome sequencing? This is a question. For the patients with residual virus nucleic acid, 265 overtreatment may do some harm to individuals.

266

\section{Conclusions}

268 Re-positive SARS-CoV-2 test after fulfilled discharge criteria is a common phenomenon. The

269

270

271

272

273

274

275

276

277

278

279

280

281

282

283

284

285

286

287

288

289

290

291

292

293

294

295

296

297

298

299

300

301

302

303

304

305

306

patients diagnosed in the early stage of illness and those with extended hospitalization were more likely to have recurrent positive results. Virus re-infection is impossible in our report, and reactivation is also unlikely, so that residual virus nucleic acid should be a reasonable explanation for this phenomenon. Under the fact that there is insufficient evidence to exclude infectivity in re-positive patients, it is better to quarantine patients after discharge for a prolonged period with repeated RT-PCR tests. It may minimize the community's potential risk, particularly in the region with relatively plenty of resources and low community infection rates, such as Macau.

\section{References}

1. Li Q, Guan X, Wu P, Wang X, Zhou L, Tong Y, Ren R, Leung KSM, Lau EHY, Wong JY, Xing X, Xiang N, Wu Y, Li C, Chen Q, Li D, Liu T, Zhao J, Liu M, Tu W, Chen C, Jin L, Yang R, Wang Q, Zhou S, Wang R, Liu H, Luo Y, Liu Y, Shao G, Li H, Tao Z, Yang Y, Deng Z, Liu B, Ma Z, Zhang Y, Shi G, Lam TTY, Wu JT, Gao GF, Cowling BJ, Yang B, Leung GM, Feng Z. Early Transmission Dynamics in Wuhan, China, of Novel Coronavirus-Infected Pneumonia. N Engl J Med 2020; 382(13): 1199-207.

2. World Health Organization. Statement on the Second Meeting of the International Health Regulations. Emergency Committee regarding the outbreak of novel coronavirus (2019-nCoV).

Available from: https://www.who.int/news/item/30-01-2020-statement-on-the-second-meeting-ofthe-international-health-regulations-(2005)-emergency-committee-regarding-the-outbreak-ofnovel-coronavirus-(2019-ncov). Accessed 30 Jan 2020.

3. World Health Organization. Coronavirus disease (COVID-19) Weekly Epidemiological Update and Weekly Operational Update, 3 November 2020. Available from: https://www.who.int/publications/m/item/weekly-epidemiological-update---3-november-2020. Accessed 3 Nov 2020.

4. Korber B, Fischer WM, Gnanakaran S, Yoon H, Theiler J, Abfalterer W, Hengartner N, Giorgi EE, Bhattacharya T, Foley B, Hastie KM, Parker MD, Partridge DG, Evans CM, Freeman TM, de Silva TI, Sheffield C-GG, McDanal C, Perez LG, Tang H, Moon-Walker A, Whelan SP, LaBranche CC, Saphire EO, Montefiori DC. Tracking Changes in SARS-CoV-2 Spike: Evidence that D614G Increases Infectivity of the COVID-19 Virus. Cell 2020; 182(4): 812-27 e19.

5. Macao SAR Government Portal. No more new cases for over two months. Available from: https://www.gov.mo/zh-hant/news/334482. Accessed 24 Jun 2020.

6. Lio CF, Cheong HH, Lei Cl, Lo IL, Lam C, Leong IH. Minimizing the risk of community spread of COVID-19 via institutional quarantine of high-risk travelers with serial viral RNA testing: A successful experience from Macao SAR, China. World J Clin Cases 2020; 8(13): 2674-8.

7. Lan L, Xu D, Ye G, Xia C, Wang S, Li Y, Xu H. Positive RT-PCR Test Results in Patients Recovered From COVID-19. JAMA 2020; 323(15): 1502-3.

Peer) reviewing PDF | (2020:11:55715:1:1:NEW 3 Feb 2021) 
307 8. Yuan J, Kou S, Liang Y, Zeng J, Pan Y, Liu L. Polymerase Chain Reaction Assays

308 Reverted to Positive in 25 Discharged Patients With COVID-19. Clin Infect Dis 2020; 71(16):

309 2230-2.

310

311

312

313

314 9. Zou Y, Wang BR, Sun L, Xu S, Kong YG, Shen LJ, Liang GT, Chen SM. The Issue of Recurrently Positive Patients Who Recovered From COVID-19 According to the Current Discharge Criteria: Investigation of Patients from Multiple Medical Institutions in Wuhan, China. $J$ Infect Dis 2020; 222(11): 1784-8.

10. Wang X, Xu H, Jiang H, Wang L, Lu C, Wei X, Liu J, Xu S. Clinical features and outcomes of discharged coronavirus disease 2019 patients: a prospective cohort study. QJM 2020; 113(9): 657-65.

11. Kang YJ. South Korea's COVID-19 Infection Status: From the Perspective of Re-positive Test Results After Viral Clearance Evidenced by Negative Test Results. Disaster medicine and public health preparedness 2020: 1-3.

320

321

322

323

324

325

326 12. Xiao AT, Tong YX, Zhang S. False-negative of RT-PCR and prolonged nucleic acid conversion in COVID-19: rather than recurrence. Journal of medical virology 2020; https://doi.org/10.1002/jmv.25855.

13. Justin W, Wee CK, Riamiza NM, Mohammad FA, Noraskhin F, Lin N. Probable Causes and Risk Factors for Positive SARS-CoV-2 Test in Recovered Patients: Evidence From Brunei Darussalam. Journal of medical virology; https://doi.org/10.1002/jmv.26199.

14. National Health Commission \& National Administration of Traditional Chinese Medicine. Diagnosis and Treatment Protocol for Novel Coronavirus Pneumonia (Trial Version 7). Chinese medical journal 2020; 133(9): 1087-95.

15. Centers for Disease Control and Prevention. Guidelines for Environmental Infection Control in Health-Care Facilities (2003). Available from:

https://www.cdc.gov/infectioncontrol/guidelines/environmental/background/air.html\#c5b. Accessed 22 Jul 2019.

16. Wikramaratna PS, Paton RS, Ghafari M, Lourenco J. Estimating the false-negative test probability of SARS-CoV-2 by RT-PCR. Euro surveillance : bulletin Europeen sur les maladies transmissibles = European communicable disease bulletin 2020; 25(50).

336 17. Huggett JF, Benes V, Bustin SA, Garson JA, Harris K, Kammel M, Kubista M, McHugh

337 TD, Moran-Gilad J, Nolan T, Pfaffl MW, Salit M, Shipley G, Vallone PM, Vandesompele J, 338 Wittwer C, Zeichhardt H. Cautionary Note on Contamination of Reagents Used for Molecular 339 Detection of SARS-CoV-2. Clinical chemistry 2020; 66(11): 1369-72.

340 18. Volz E, Hill V, McCrone JT, Price A, Jorgensen D, O'Toole Á, Southgate J, Johnson R, Jackson B, Nascimento FF, Rey SM, Nicholls SM, Colquhoun RM, da Silva Filipe A, Shepherd J, Pascall DJ, Shah R, Jesudason N, Li K, Jarrett R, Pacchiarini N, Bull M, Geidelberg L, Siveroni I, Goodfellow I, Loman NJ, Pybus OG, Robertson DL, Thomson EC, Rambaut A, Connor TR. Evaluating the Effects of SARS-CoV-2 Spike Mutation D614G on Transmissibility and Pathogenicity. Cell 2021; 184(1): 64-75.e11.

346 19. To KK, Hung IF, Ip JD, Chu AW, Chan WM, Tam AR, Fong CH, Yuan S, Tsoi HW, Ng AC, Lee LL, Wan P, Tso E, To WK, Tsang D, Chan KH, Huang JD, Kok KH, Cheng VC, Yuen KY. COVID-19 re-infection by a phylogenetically distinct SARS-coronavirus-2 strain confirmed by whole genome sequencing. Clin Infect Dis 2020; https://doi.org/10.1093/cid/ciaa1275. 20. Liu WD, Chang SY, Wang JT, Tsai MJ, Hung CC, Hsu CL, Chang SC. Prolonged virus shedding even after seroconversion in a patient with COVID-19. J Infect 2020; 81(2): 318-56.

352

353

354

355 21. Wölfel R, Corman VM, Guggemos W, Seilmaier M, Zange S, Müller MA, Niemeyer D, Jones TC, Vollmar P, Rothe C. Virological assessment of hospitalized patients with COVID2019. Nature 2020; https://doi.org/10.1038/s41586-020-2196-x. 


\section{Table 1 (on next page)}

The clinical characteristics of re-positive group and negative group 
Table 1. The clinical characteristics of re-positive group and negative group

\begin{tabular}{|c|c|c|c|c|}
\hline \multirow{2}{*}{ Clinical characteristics } & \multicolumn{2}{|c|}{ Groups } & \multirow{2}{*}{$\begin{array}{l}\chi^{2} / Z \\
\text { value }\end{array}$} & \multirow{2}{*}{$\begin{array}{c}\boldsymbol{P} \\
\text { value }\end{array}$} \\
\hline & Re-positive group & Negative group & & \\
\hline Age, median (IQR) & $28.5(18.3-42.3)$ & $28(20.0-44.0)$ & -0.626 & 0.542 \\
\hline \multicolumn{5}{|l|}{ Gender, No. (\%) } \\
\hline Male $(n=23)$ & $6(26.1)$ & $17(73.9)$ & \multirow[t]{2}{*}{---} & \multirow[t]{2}{*}{0.149} \\
\hline Female $(\mathrm{n}=12)$ & $6(50.0)$ & $6(50.0)$ & & \\
\hline \multicolumn{5}{|l|}{ Nationality, No. (\%) } \\
\hline Chinese $(n=23)$ & $8(34.8)$ & $15(65.2)$ & \multirow[t]{7}{*}{11.843} & \multirow[t]{7}{*}{0.066} \\
\hline Filipino $(n=3)$ & $3(100)$ & $0(0)$ & & \\
\hline Indonesian $(\mathrm{n}=3)$ & $0(0)$ & $3(100)$ & & \\
\hline Portuguese $(n=3)$ & $0(0)$ & $3(100)$ & & \\
\hline Australian $(n=1)$ & $0(0)$ & $1(100)$ & & \\
\hline Korean $(n=1)$ & $1(100)$ & $0(0)$ & & \\
\hline Spanish $(\mathrm{n}=1)$ & $0(0)$ & $1(100)$ & & \\
\hline \multicolumn{5}{|l|}{ Travel history, No. (\%) } \\
\hline England $(\mathrm{n}=16)$ & $5(31.3)$ & $11(68.8)$ & \multirow[t]{9}{*}{8.129} & \multirow[t]{9}{*}{0.421} \\
\hline Philippines $(n=5)$ & $3(60.0)$ & $2(40.0)$ & & \\
\hline Portugal $(n=4)$ & $1(25.0)$ & $3(75.0)$ & & \\
\hline America $(n=3)$ & $2(66.7)$ & $1(33.3)$ & & \\
\hline Indonesia $(\mathrm{n}=3)$ & $0(0)$ & $3(100)$ & & \\
\hline Cambodia $(\mathrm{n}=1)$ & $0(0)$ & $1(100)$ & & \\
\hline Ireland $(n=1)$ & $1(100)$ & $0(0)$ & & \\
\hline Spain $(n=1)$ & $0(0)$ & $1(100)$ & & \\
\hline Thailand $(\mathrm{n}=1)$ & $0(0)$ & $1(100)$ & & \\
\hline \multicolumn{5}{|c|}{ S/S before hospitalization, No. (\%) } \\
\hline Yes $(n=28)$ & $11(39.3)$ & $17(60.7)$ & \multirow[t]{2}{*}{---} & \multirow[t]{2}{*}{0.217} \\
\hline No $(n=7)$ & $1(14.3)$ & $6(85.7)$ & & \\
\hline \multicolumn{5}{|c|}{ Classification when admission, No. (\%) } \\
\hline Asymptomatic type $(n=3)$ & $0(0)$ & $3(100)$ & \multirow[t]{3}{*}{1.712} & \multirow[t]{3}{*}{0.425} \\
\hline Mild type $(n=16)$ & $6(37.5)$ & $10(62.5)$ & & \\
\hline Moderate type $(n=16)$ & $6(37.5)$ & $10(62.5)$ & & \\
\hline \multicolumn{5}{|c|}{ Classification during hospitalization, } \\
\hline \multicolumn{5}{|c|}{ No. $(\%)$} \\
\hline Asymptomatic type $(n=3)$ & $0(0)$ & $3(100)$ & \multirow[t]{4}{*}{3.061} & \multirow[t]{4}{*}{0.382} \\
\hline Mild type $(n=12)$ & $6(50.0)$ & $6(50.0)$ & & \\
\hline Moderate type $(n=17)$ & $5(29.4)$ & $12(70.6)$ & & \\
\hline Severe to critical $(\mathrm{n}=3)$ & $1(33.3)$ & $2(66.7)$ & & \\
\hline \multicolumn{5}{|l|}{ Past medical history, No. (\%) } \\
\hline Yes $(n=6)$ & $1(16.7)$ & $5(83.3)$ & --- & 0.311 \\
\hline No $(n=29)$ & $11(37.9)$ & $18(62.1)$ & & \\
\hline Laboratory test, median (IQ & & & & \\
\hline Highest CRP & $0.31(0.11-1.13)$ & $0.32(0.05-1.88)$ & -0.243 & 0.817 \\
\hline Highest PCT & $0.05(0.03-0.06)$ & $0.03(0.03-0.06)$ & -1.078 & 0.290 \\
\hline CT when admission, No. (\% & & & & \\
\hline Normal $(n=19)$ & $6(31.6)$ & $13(68.4)$ & --- & 0.495 \\
\hline Pneumonia $(n=16)$ & $6(37.5)$ & $10(62.5)$ & & \\
\hline
\end{tabular}


Table 2 (on next page)

The main treatment used in re-positive group and negative group 
Table 2. The main treatment used in re-positive group and negative group

\begin{tabular}{|c|c|c|c|}
\hline \multirow{2}{*}{ Main treatment } & \multicolumn{2}{|c|}{ Groups } & \multirow{2}{*}{$\begin{array}{c}\boldsymbol{P} \\
\text { value }\end{array}$} \\
\hline & Re-positive group & Negative group & \\
\hline \multicolumn{4}{|c|}{ Lopinavir / ritonavir, No. (\%) } \\
\hline 2 weeks $(n=13)$ & $3(23.1)$ & $10(76.9)$ & \multirow[t]{2}{*}{0.243} \\
\hline 3 weeks $(\mathrm{n}=22)$ & $9(40.9)$ & $13(59.1)$ & \\
\hline \multicolumn{4}{|l|}{ Interferon, No. (\%) } \\
\hline Used $(\mathrm{n}=12)$ & $4(33.3)$ & $8(66.7)$ & \multirow[t]{2}{*}{0.618} \\
\hline Not used $(n=23)$ & $8(34.8)$ & $15(65.2)$ & \\
\hline \multicolumn{4}{|l|}{ Azithromycin, №. (\%) } \\
\hline Used $(n=32)$ & $12(37.5)$ & $20(62.5)$ & \multirow[t]{2}{*}{0.271} \\
\hline Not used $(\mathrm{n}=3)$ & $0(0)$ & $3(100)$ & \\
\hline \multicolumn{4}{|l|}{ Levofloxacin, No. (\%) } \\
\hline Used $(n=11)$ & $4(36.4)$ & $7(63.6)$ & \multirow[t]{2}{*}{0.576} \\
\hline Not used $(n=24)$ & $8(33.3)$ & $16(66.7)$ & \\
\hline \multicolumn{4}{|c|}{ Methylprednisolone, No. (\%) } \\
\hline Used $(n=2)$ & $1(50.0)$ & $1(50.0)$ & \multirow[t]{2}{*}{0.545} \\
\hline Not used $(n=33)$ & $11(33.3)$ & $22(66.7)$ & \\
\hline \multicolumn{4}{|c|}{ Hydroxychloroquine, No. (\%) } \\
\hline Used $(n=7)$ & $2(28.6)$ & $5(71.4)$ & \multirow[t]{2}{*}{0.547} \\
\hline Not used $(n=28)$ & $10(35.7)$ & $18(64.3)$ & \\
\hline
\end{tabular}




\section{Table 3 (on next page)}

Timeline of disease progression in re-positive group and negative group 
Table 3. Timeline of disease progression in re-positive group and negative group

\begin{tabular}{|c|c|c|c|c|}
\hline \multirow{2}{*}{ Timeline of disease progression, days } & \multicolumn{2}{|c|}{ Groups } & \multirow{2}{*}{$\begin{array}{c}\boldsymbol{Z} \\
\text { value }\end{array}$} & \multirow{2}{*}{$\begin{array}{c}\boldsymbol{P} \\
\text { value }\end{array}$} \\
\hline & Re-positive group & Negative group & & \\
\hline S/S onset to diagnosis, median (IQR) & $2.0(1.0-5.0)$ & $4.0(1.5-11.5)$ & -1.445 & 0.154 \\
\hline S/S onset to hospital discharge, median (IQR) & $43.0(26.0-55.0)$ & $35.0(27.5-41.5)$ & -1.177 & 0.249 \\
\hline Diagnosis to hospital discharge, median (IQR) & $38.0(25.3-50.5)$ & $27.0(20.0-38.0)$ & -1.827 & 0.069 \\
\hline
\end{tabular}

